\title{
Evaluation Method of Weapons Developing program based on Program-ability satisfied degree
}

\author{
Xiao-pan Wang ${ }^{1, a}$, Ya-jie Dou ${ }^{1, b}$ and Qing-song Zhao ${ }^{1, c}$, Sheng-ping ji ji $^{2, d}$ \\ ${ }^{1}$ College of Information System and Management, National University of Defense Technology, \\ Changsha, 410005, China. \\ ${ }^{2}$ Section 89, Group 93062, Jilin, 132102, China. \\ anudt_wxp@163.com, bdouyajie_nudt@163.com, czhaoqingsong@nudt.edu.cn, d296007898@qq.c \\ om
}

Keywords: the satisfied degree, interval number, the improved TOPSIS method.

\begin{abstract}
In the top-level design of the construction of weapons, how to evaluate alternatives scientifically and accurately, and select the best scheme is a very important job. In this paper, we use 'the Program-ability satisfied degree' to measure the satisfied degree of a weapons developing program to function needs and provide a reference for decision makers to evaluate alternatives and choose the best one among them. At first, based on expert scoring method, we do paired comparison of the satisfied degree of weapons developing alternatives to function needs, then, by using the optimized mathematical model to deal with data of expert scoring, we get the satisfied degree of alternatives, at last, we use The improved TOPSIS method to sort the alternatives according to the size of the satisfied degree. The calculating example shows the feasibility of the method.
\end{abstract}

\section{Introduction}

The evaluation and selection of weapons developing program is an important link of the demonstration of weapon equipment construction, after the comprehensive demonstration project, decision makers should choose the best one among alternatives. Modern weapons have characteristics of high content of science and technology, large scale, and it's development and production cost a large of money and time, which require the choice of decision makers should be scientific and accurate, the traditional way of thinking and decision making is no longer applicable[1].

Factors to be considered are complicated and have multiple levels when decision makers choose the best one among alternatives. In many factors, whether the possible program can meet the needs of function and how much the satisfied degree is are the most fundamental and important factors. In this paper, we use 'the Program - ability satisfaction degree' to measure the satisfied degree of a weapon building program to function needs and provide a reference for decision makers to evaluate alternatives and choose the best one among them.

The assessment of the satisfied degree is to assess the possibility of a program meet the function needs when the level of the needs of function is known. Up to now, there are only a few mature methods dealing with the problem about the assessment of the ability to meet the needs of function. Shuyu gave a method to assess the ability of architecture to meet the need of System function. In the paper, satisfied degree of system function is defined to be including proximity and relativity, and GCA-TOPSIS is used to build the model. This method is complicated and has a high requirement to data, which make the method can only be used in ideal condition[2].

In the actual engineering practice, we usually can't get exactly and completed data. In most cases decisions are made through the method of expert review. When the numbers of alternatives and ability index are large, experts can't make reliable decision. To deal with this problem, in this paper, we use 'the Program-ability satisfaction degree' to measure the satisfied degree of a weapon building program to function needs and evaluate alternatives. The method has the following steps: 
a、 Design an expert questionnaire and do paired comparison of the satisfied degree of weapon building programs to function needs.

b、 By using the optimized mathematical model to deal with data of Expert scoring, we get the satisfied degree of alternatives.

c、 We use the improved TOPSIS method to sort the alternatives according to the size of the satisfied degree.

\section{Data collection by questionnaire survey}

Because experts can only get a few information, it's difficult to get exact amount of the satisfied degree. In this paper, we use questionnaire to get experts'Personal preference about which possible program has bigger satisfied degree by letting experts do paired comparison of alternatives.

We denote by $A^{*}=\left\{A_{t}, t=1,2, \ldots \mathrm{m}\right\}$ the vector of alternatives, the vector of the needs of function is represented by the vector $C^{*}=\left\{C_{u}, u=1,2, \ldots \mathrm{n}\right\}$, the satisfied degree of $A_{t}$ to $C_{u}$ is represented by $\operatorname{ASL}\left(A_{t}, C_{u}\right)$.

Take the paired comparison of $A^{*}=\left\{A_{t}, t=1,2, \ldots \mathrm{m}\right\}$ into consideration, we get paired comparison matrix by comparing the size of the satisfied degree of $A_{t}$ to $C_{u}$ and $A_{h}$ to $C_{u}, t, h \in N^{+}, t, h \leq \mathrm{m}$. The score of the comparison can be represented by $a_{t h}, a_{t h}$ can score integers from 1 to $9[3]$.

The meaning of $a_{t h}$ is as follow:

a、 $a_{t h}=1$ : to the function requirement of $C_{u}, A_{t}$ is the same as $A_{h}$,

b、 $a_{t h}=3$ : to the function requirement of $C_{u}, A_{t}$ is fairly better than $A_{h}$,

c、 $a_{t h}=5$ : to the function requirement of $C_{u}, A_{t}$ is a litter better than $A_{h}$,

d、 $a_{t h}=7$ : to the function requirement of $C_{u}, A_{t}$ is much better than $A_{h}$,

e、 $a_{t h}=9$ : to the function requirement of $C_{u}, A_{t}$ is most better than $A_{h}$,

And the other numbers 2, 4, 6 and 8 are used for the supplementary.

Through questionnaire survey, we can get $\mathrm{n}$ m-dimensional paired comparison matrixes as follow:

$$
\mathrm{T}\left(c_{j}\right)_{m \times m}=\left[\begin{array}{cccc}
1 & a_{12} & \cdots & a_{1 m} \\
1 / a_{12} & 1 & \cdots & a_{2 m} \\
\vdots & \vdots & \ddots & \vdots \\
1 / a_{1 m} & 1 / a_{2 m} & \cdots & 1
\end{array}\right]
$$

\section{Optimized mathematical model}

We construct optimized mathematical model by the method of linear programming to deal with data of expert scoring and get the satisfied degree. Because the assessment is complicated and uncertain, we can't get exactly numbers of the satisfied degree but interval numbers, which is as follow[4]:

$$
\operatorname{ASL}\left(A_{t}, C_{u}\right)=\left[L_{t}^{C_{u}-}, L_{t}^{C_{u}+}\right]
$$

The upper limit and lower limit of $\operatorname{ASL}\left(A_{t}, C_{u}\right)$ are $L_{t}^{C_{u}{ }^{+}}$and $L_{t}^{C_{u}-}$, which have constraint as follow:

$$
0 \leq L_{t}^{C_{u}-} \leq L_{t}^{C_{u}+} \leq 1
$$

It's obviously that $\operatorname{ASL}\left(A_{t}, C_{u}\right) \in[0,1]$. If $L_{t}^{C_{u}{ }^{-}}=L_{t}{ }^{C_{u}{ }^{+}}, A S L\left(A_{t}, C_{u}\right)$ become an exact number. The width of $\operatorname{ASL}\left(A_{t}, C_{u}\right)$ can be defined as follow:

$$
w\left(\operatorname{ASL}\left(A_{t}, C_{u}\right)\right)=L_{t}^{C_{u}+}-L_{t}^{C_{u}-}
$$


We assume that $\forall L_{t}^{C_{u}} \in\left[L_{t}^{C_{u}-}, L_{t}^{C_{u}+}\right]$ satisfy the equation $\sum_{t=1}^{k} L_{t}^{C_{u}}=1$. So we can get:

$$
\begin{array}{ll}
L_{t}^{C_{u}+}+L_{1}^{C_{u}-}+\cdots+L_{t-1}{ }^{C_{u}-}+L_{t+1}{ }^{C_{u}-}+\cdots+L_{k} C_{u^{-}} \leq 1 & \forall t=1,2, \cdots, k, \\
L_{t}^{C_{u}-}+L_{1}^{C_{u}+}+\cdots+L_{t-1}^{-C_{u}+}+L_{t+1}{ }^{C_{u}+}+\cdots+L_{k}{ }^{C_{u}+} \geq 1 & \forall t=1,2, \cdots, k .
\end{array}
$$

We can get interval ratio method as follow:

$$
\operatorname{TSL}\left(T_{t}, C_{u}\right) / \operatorname{TSL}\left(T_{h}, C_{u}\right)=\left[L_{t}{ }^{C_{u}-} / L_{h}{ }^{C_{u}+}, L_{t}{ }^{C_{u}+} / L_{h}{ }^{C_{u}-}\right]
$$

The $a_{t h}$ we get through expert scoring belong to Interval ratio $\operatorname{ASL}\left(A_{t}, C_{u}\right) / \operatorname{ASL}\left(A_{h}, C_{u}\right)$, which can be expressed as:

$$
\begin{aligned}
& a_{t h} \in\left[L_{t}^{C_{u}-} / L_{h}^{C_{u}+}, L_{t}^{C_{u}+} / L_{h}^{C_{u}-}\right] \\
& \Leftrightarrow L_{t}^{C_{u}-} / L_{h}^{C_{u}+} \leq a_{t h} \leq L_{t}^{C_{u}+} / L_{h}^{C_{u}-} \\
& \Leftrightarrow\left\{\begin{array}{l}
L_{t}^{C_{u}-}-a_{t h} L_{h}^{C_{u}+} \leq 0, \\
L_{t}^{C_{u}+}-a_{t h} L_{h} C_{u}-\geq 0, \\
L_{t}^{C_{u}-} \geq \varepsilon .
\end{array}\right.
\end{aligned}
$$

$\varepsilon$ represents a positive number which is very small.

From the above, we can get optimized mathematical model as follow[5]:

$$
\begin{aligned}
& \min _{L_{t}^{C_{u}^{+}, L_{t}^{C_{u}}}} I^{1}\left(A S L\left(T, C_{u}\right)^{*}\right)=\frac{1}{k} \sum_{t=1}^{k}\left(L_{t}^{C_{u}+}-L_{t}^{C_{u}-}\right) \\
& \text { s.t. } L_{t}^{C_{u}{ }^{+}}+L_{1}^{C_{u}-}+\ldots+L_{i-1}{ }^{C_{u}-}+L_{i+1}{ }^{C_{u}-}+\ldots+L_{k} C^{C^{-}} \leq 1 \quad \forall t=1,2, \ldots k \text {, } \\
& L_{t}^{C^{C^{-}}}+L_{1}^{C_{u}+}+\ldots+L_{t-1}{ }^{C_{u}+}+L_{t+1}{ }^{C_{u}+}+\ldots+L_{k}{ }^{C_{u}+} \geq 1 \quad \forall t=1,2, \ldots k, \\
& L_{t}^{C^{-}-}-a_{t h} L_{h}{ }^{C_{u}{ }^{+}} \leq 0 \quad \forall(t=1,2, \ldots k, h>t), \\
& L_{t}^{C_{u}{ }^{+}-a_{t h}} L_{h}^{C_{u^{-}}} \geq 0 \quad \forall(t=1,2, \ldots k, h>t), \\
& L_{t}^{C_{u}-} \geq \varepsilon \quad \forall t=1,2, \ldots k \text {, } \\
& L_{t}^{C_{u}+}-L_{t}^{C_{u}-} \geq 0 \quad \forall t=1,2, \ldots k .
\end{aligned}
$$

After we get the satisfied degree, we can weigh the result according to the difference of the importance of the function needs.

\section{Sorting alternatives based on improved TOPSIS}

As a classical and being in common used MCDM method which uses Cardinality information, The TOPSIS method is now successfully used in many fields, such as material selection, energy projects and supply chain management. The main idea of TOPSIS is finding the positive ideal solution (PIS) and the negative ideal solution (NIS), and uses the proportion of the distance of an alternative to the positive ideal solution and the distance of an alternative to the negative ideal solution to assess the alternatives.

After we get the satisfied degree, we use the improved TOPSIS method, which uses interval number in the TOPSIS method, to assess alternatives. The basic steps are as follow[6]:

a、 Find the positive ideal solution (PIS) and the negative ideal solution (NIS).

b、 Compute the distance of an alternative to the positive ideal solution and the distance of an alternative to the negative ideal solution.

The distance of the $\mathrm{t}^{\text {th }}$ alternative $A_{t}$ to the positive ideal solution is: 


$$
d_{t}^{*}=\sqrt{\sum_{u=1}^{n}\left(L_{t}^{C_{u}+}-x_{u}^{*}\right)^{2}+\sum_{j=1}^{n}\left(L_{t}^{C_{u}-}-x_{u}^{*}\right)^{2}}, u=1, \cdots, \mathrm{n}
$$

The distance of $A_{t}$ to the negative ideal solution is:

$$
d_{t}^{0}=\sqrt{\sum_{u=1}^{n}\left(L_{t}^{C_{u}+}-x_{u}^{0}\right)^{2}+\sum_{j=1}^{n}\left(L_{t}^{C_{u}-}-x_{u}^{0}\right)^{2}}, u=1, \cdots, \mathrm{n}
$$

c、 Compute the relative closeness degree as follow.

$$
C_{t}^{*}=d_{t}^{0} /\left(d_{t}^{0}+d_{t}^{*}\right), t=1, \cdots, m
$$

d、 Sorting alternatives from good to bad according to $C_{t}^{*}$, the greater the $C_{t}^{*}$ is ,the better the alternative is.

\section{Example and analysis}

We suppose that there are ten alternatives and four function needs. To every function need, we do paired comparison of the satisfied degree of all alternatives, and build paired comparison matrixes, then by using the optimization model, we get the satisfied degree. A1 to A10 represent ten alternatives, C1 to C4 represent four function needs. The paired comparison matrixes of alternatives of every function need is showed as follow:

Table 1 The paired comparison matrix of C1

\begin{tabular}{lllllllllll}
\hline & $\mathrm{A}_{1}$ & $\mathrm{~A}_{2}$ & $\mathrm{~A}_{3}$ & $\mathrm{~A}_{4}$ & $\mathrm{~A}_{5}$ & $\mathrm{~A}_{6}$ & $\mathrm{~A}_{7}$ & $\mathrm{~A}_{8}$ & $\mathrm{~A}_{9}$ & $\mathrm{~A}_{10}$ \\
\hline $\mathrm{A}_{1}$ & 1 & 2 & 2 & 2 & 3 & 3 & 3 & 9 & 9 & 9 \\
$\mathrm{~A}_{2}$ & $1 / 2$ & 1 & 1 & 1 & 2 & 2 & 2 & 5 & 5 & 5 \\
$\mathrm{~A}_{3}$ & $1 / 2$ & 1 & 1 & 1 & 2 & 2 & 2 & 5 & 5 & 5 \\
$\mathrm{~A}_{4}$ & $1 / 2$ & 1 & 1 & 1 & 2 & 2 & 2 & 5 & 5 & 5 \\
$\mathrm{~A}_{5}$ & $1 / 3$ & $1 / 2$ & $1 / 2$ & $1 / 2$ & 1 & 1 & 1 & 3 & 3 & 3 \\
$\mathrm{~A}_{6}$ & $1 / 3$ & $1 / 2$ & $1 / 2$ & $1 / 2$ & 1 & 1 & 1 & 3 & 3 & 3 \\
$\mathrm{~A}_{7}$ & $1 / 3$ & $1 / 2$ & $1 / 2$ & $1 / 2$ & 1 & 1 & 1 & 3 & 3 & 3 \\
$\mathrm{~A}_{8}$ & $1 / 9$ & $1 / 5$ & $1 / 5$ & $1 / 5$ & $1 / 3$ & $1 / 3$ & $1 / 3$ & 1 & 1 & 1 \\
$\mathrm{~A}_{9}$ & $1 / 9$ & $1 / 5$ & $1 / 5$ & $1 / 5$ & $1 / 3$ & $1 / 3$ & $1 / 3$ & 1 & 1 & 1 \\
$\mathrm{~A}_{10}$ & $1 / 9$ & $1 / 5$ & $1 / 5$ & $1 / 5$ & $1 / 3$ & $1 / 3$ & $1 / 3$ & 1 & 1 & 1 \\
\hline
\end{tabular}

Table 2 The paired comparison matrix of C2

\begin{tabular}{lllllllllll}
\hline & $\mathrm{A} 1$ & $\mathrm{~A} 2$ & $\mathrm{~A} 3$ & $\mathrm{~A} 4$ & $\mathrm{~A} 5$ & $\mathrm{~A} 6$ & $\mathrm{~A} 7$ & $\mathrm{~A} 8$ & $\mathrm{~A} 9$ & $\mathrm{~A} 10$ \\
\hline $\mathrm{A} 1$ & 1 & 1 & 1 & 1 & 3 & 3 & 3 & 9 & 9 & 9 \\
$\mathrm{~A} 2$ & 1 & 1 & 1 & 1 & 3 & 3 & 3 & 9 & 9 & 9 \\
$\mathrm{~A} 3$ & 1 & 1 & 1 & 1 & 3 & 3 & 3 & 9 & 9 & 9 \\
$\mathrm{~A} 4$ & 1 & 1 & 1 & 1 & 3 & 3 & 3 & 9 & 9 & 9 \\
$\mathrm{~A} 5$ & $1 / 3$ & $1 / 3$ & $1 / 3$ & $1 / 3$ & 1 & 1 & 1 & 3 & 3 & 3 \\
$\mathrm{~A} 6$ & $1 / 3$ & $1 / 3$ & $1 / 3$ & $1 / 3$ & 1 & 1 & 1 & 3 & 3 & 3 \\
$\mathrm{~A} 7$ & $1 / 3$ & $1 / 3$ & $1 / 3$ & $1 / 3$ & 1 & 1 & 1 & 3 & 3 & 3 \\
$\mathrm{~A} 8$ & $1 / 9$ & $1 / 9$ & $1 / 9$ & $1 / 9$ & $1 / 3$ & $1 / 3$ & $1 / 3$ & 1 & 1 & 1 \\
$\mathrm{~A} 9$ & $1 / 9$ & $1 / 9$ & $1 / 9$ & $1 / 9$ & $1 / 3$ & $1 / 3$ & $1 / 3$ & 1 & 1 & 1 \\
$\mathrm{~A} 10$ & $1 / 9$ & $1 / 9$ & $1 / 9$ & $1 / 9$ & $1 / 3$ & $1 / 3$ & $1 / 3$ & 1 & 1 & 1 \\
\hline
\end{tabular}

Table 3 The paired comparison matrix of C3

\begin{tabular}{lllllllllll}
\hline & $\mathrm{A} 1$ & $\mathrm{~A} 2$ & $\mathrm{~A} 3$ & $\mathrm{~A} 4$ & $\mathrm{~A} 5$ & $\mathrm{~A} 6$ & $\mathrm{~A} 7$ & $\mathrm{~A} 8$ & $\mathrm{~A} 9$ & $\mathrm{~A} 10$ \\
\hline $\mathrm{A} 1$ & 1 & 1 & 1 & 1 & 4 & 4 & 4 & 8 & 8 & 8 \\
$\mathrm{~A} 2$ & 1 & 1 & 1 & 1 & 4 & 4 & 4 & 8 & 8 & 8 \\
$\mathrm{~A} 3$ & 1 & 1 & 1 & 1 & 4 & 4 & 4 & 8 & 8 & 8 \\
$\mathrm{~A} 4$ & 1 & 1 & 1 & 1 & 4 & 4 & 4 & 8 & 8 & 8 \\
$\mathrm{~A} 5$ & $1 / 4$ & $1 / 4$ & $1 / 4$ & $1 / 4$ & 1 & 1 & 1 & 2 & 2 & 2 \\
$\mathrm{~A} 6$ & $1 / 4$ & $1 / 4$ & $1 / 4$ & $1 / 4$ & 1 & 1 & 1 & 2 & 2 & 2 \\
$\mathrm{~A} 7$ & $1 / 4$ & $1 / 4$ & $1 / 4$ & $1 / 4$ & 1 & 1 & 1 & 2 & 2 & 2 \\
$\mathrm{~A} 8$ & $1 / 8$ & $1 / 8$ & $1 / 8$ & $1 / 8$ & $1 / 2$ & $1 / 2$ & $1 / 2$ & 1 & 1 & 1 \\
$\mathrm{~A} 9$ & $1 / 8$ & $1 / 8$ & $1 / 8$ & $1 / 8$ & $1 / 2$ & $1 / 2$ & $1 / 2$ & 1 & 1 & 1 \\
$\mathrm{~A} 10$ & $1 / 8$ & $1 / 8$ & $1 / 8$ & $1 / 8$ & $1 / 2$ & $1 / 2$ & $1 / 2$ & 1 & 1 & 1 \\
\hline
\end{tabular}


Table 4 The paired comparison matrix of C4

\begin{tabular}{lllllllllll}
\hline & $\mathrm{A} 1$ & $\mathrm{~A} 2$ & $\mathrm{~A} 3$ & $\mathrm{~A} 4$ & $\mathrm{~A} 5$ & $\mathrm{~A} 6$ & $\mathrm{~A} 7$ & $\mathrm{~A} 8$ & $\mathrm{~A} 9$ & $\mathrm{~A} 10$ \\
\hline $\mathrm{A} 1$ & 1 & 3 & 3 & 3 & 2 & 2 & 2 & 9 & 9 & 9 \\
$\mathrm{~A} 2$ & $1 / 3$ & 1 & 1 & 1 & $1 / 2$ & $1 / 2$ & $1 / 2$ & 3 & 3 & 3 \\
$\mathrm{~A} 3$ & $1 / 3$ & 1 & 1 & 1 & $1 / 2$ & $1 / 2$ & $1 / 2$ & 3 & 3 & 3 \\
$\mathrm{~A} 4$ & $1 / 3$ & 1 & 1 & 1 & $1 / 2$ & $1 / 2$ & $1 / 2$ & 3 & 3 & 3 \\
$\mathrm{~A} 5$ & $1 / 2$ & 2 & 2 & 2 & 1 & 1 & 1 & 5 & 5 & 5 \\
$\mathrm{~A} 6$ & $1 / 2$ & 2 & 2 & 2 & 1 & 1 & 1 & 5 & 5 & 5 \\
$\mathrm{~A} 7$ & $1 / 2$ & 2 & 2 & 2 & 1 & 1 & 1 & 5 & 5 & 5 \\
$\mathrm{~A} 8$ & $1 / 9$ & $1 / 3$ & $1 / 3$ & $1 / 3$ & $1 / 5$ & $1 / 5$ & $1 / 5$ & 1 & 1 & 1 \\
$\mathrm{~A} 9$ & $1 / 9$ & $1 / 3$ & $1 / 3$ & $1 / 3$ & $1 / 5$ & $1 / 5$ & $1 / 5$ & 1 & 1 & 1 \\
$\mathrm{~A} 10$ & $1 / 9$ & $1 / 3$ & $1 / 3$ & $1 / 3$ & $1 / 5$ & $1 / 5$ & $1 / 5$ & 1 & 1 & 1 \\
\hline
\end{tabular}

Table 5 The interval satisfied degree

\begin{tabular}{lllllllll}
\hline & C1L & C1R & C2L & C2R & C3L & C3R & C4L & C4R \\
\hline A1 & 0.1259 & 0.1399 & 0.0937 & 0.0938 & 0.0976 & 0.0976 & 0.1259 & 0.1399 \\
A2 & 0.0699 & 0.0699 & 0.0937 & 0.0938 & 0.0976 & 0.0976 & 0.035 & 0.042 \\
A3 & 0.0699 & 0.0699 & 0.0937 & 0.0938 & 0.0976 & 0.0976 & 0.035 & 0.042 \\
A4 & 0.0699 & 0.0699 & 0.0937 & 0.0938 & 0.0976 & 0.0976 & 0.035 & 0.042 \\
A5 & 0.035 & 0.042 & 0.0312 & 0.0313 & 0.0244 & 0.0244 & 0.0699 & 0.0699 \\
A6 & 0.035 & 0.042 & 0.0312 & 0.0313 & 0.0244 & 0.0244 & 0.0699 & 0.0699 \\
A7 & 0.035 & 0.042 & 0.0312 & 0.0313 & 0.0244 & 0.0244 & 0.0699 & 0.0699 \\
A8 & 0.014 & 0.014 & 0.0104 & 0.0104 & 0.0122 & 0.0122 & 0.014 & 0.014 \\
A9 & 0.014 & 0.014 & 0.0104 & 0.0104 & 0.0122 & 0.0122 & 0.014 & 0.014 \\
A10 & 0.014 & 0.014 & 0.0104 & 0.0104 & 0.0122 & 0.0122 & 0.014 & 0.014 \\
\hline
\end{tabular}

Table 6 The weighted interval satisfied degree

\begin{tabular}{lllllllll}
\hline & C1L & C1R & C2L & C2R & C3L & C3R & C4L & C4R \\
\hline A1 & 0.0315 & 0.035 & 0.0234 & 0.0234 & 0.0244 & 0.0244 & 0.0315 & 0.035 \\
A2 & 0.0175 & 0.0175 & 0.0234 & 0.0234 & 0.0244 & 0.0244 & 0.0087 & 0.0105 \\
A3 & 0.0175 & 0.0175 & 0.0234 & 0.0234 & 0.0244 & 0.0244 & 0.0087 & 0.0105 \\
A4 & 0.0175 & 0.0175 & 0.0234 & 0.0234 & 0.0244 & 0.0244 & 0.0087 & 0.0105 \\
A5 & 0.0087 & 0.0105 & 0.0078 & 0.0078 & 0.0061 & 0.0061 & 0.0175 & 0.0175 \\
A6 & 0.0087 & 0.0105 & 0.0078 & 0.0078 & 0.0061 & 0.0061 & 0.0175 & 0.0175 \\
A7 & 0.0087 & 0.0105 & 0.0078 & 0.0078 & 0.0061 & 0.0061 & 0.0175 & 0.0175 \\
A8 & 0.0035 & 0.0035 & 0.0026 & 0.0026 & 0.003 & 0.003 & 0.0035 & 0.0035 \\
A9 & 0.0035 & 0.0035 & 0.0026 & 0.0026 & 0.003 & 0.003 & 0.0035 & 0.0035 \\
A10 & 0.0035 & 0.0035 & 0.0026 & 0.0026 & 0.003 & 0.003 & 0.0035 & 0.0035 \\
\hline
\end{tabular}

In the tables, $\mathrm{L}$ represents the upper limit of interval number, and $\mathrm{R}$ represents lower limit of interval number.

Then use the improved TOPSIS method to sort the alternatives according to the size of the satisfied degree. The positive ideal solution (PIS) and the negative ideal solution (NIS) of the TOPSIS method are showed in table 8, and table 9. The sorting of alternatives is showed in table 10.

Table 7 The distance of alternatives to the positive ideal solution

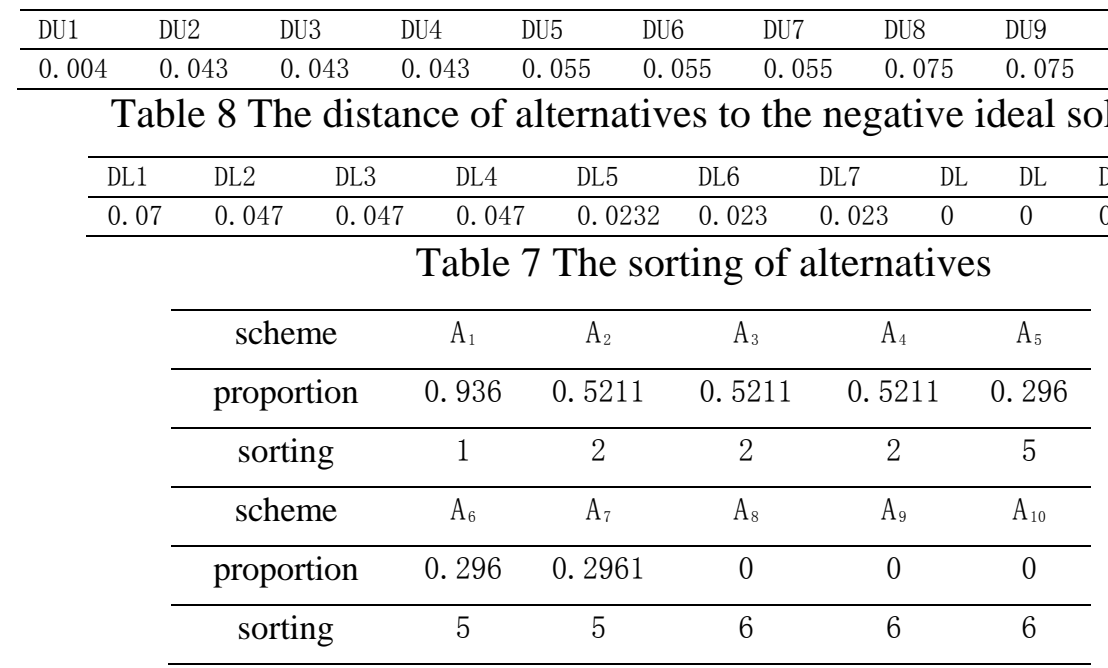


It can be seen from tables above:

a、 Scheme one is the best alternative, which has the largest satisfied degree.

b、Scheme eight, scheme nine, scheme ten have the minimum satisfied degree, which are the worst scheme.

c、The satisfied degree of scheme two, scheme three, scheme four to function need one and function need four is less than scheme one, the optimization of the three alternatives should be aimed at the two function needs.

\section{Conclusions}

The method we present in this paper is a combination of qualitative and quantitative. Based on the expert scoring method, we use the optimized mathematical model to deal with data of expert scoring and get the satisfied degree of alternatives. This method deal with the problem in decision-making process that there are few information but too many alternatives and function needs to be taken into consideration, besides, we cover the shortage that decision making is mainly depended on expert review. The satisfied degree is an abstract concept, this method can also be applied to the consideration of other factors, such as cost, cost and risk. In practical application of this method, the main difficulty is collecting data through expert questionnaire.

\section{Acknowledgements}

The research work was supported by National Natural Science Foundation of China under Grant No. 71331008 and 71201168.

\section{References}

[1] Meng Xian-feng, Zhang Yu-zhu. Study of Weapons Developing Alter-natives Decision Making based on Utility Analysis. Fire Control and Command Control, 32(2), pp.62-65, 2007.

[2] Shu Y. Research on the method and application of the architecture modeling of weapon system-of-Systems based on cability requirement. Changsha: National University of Defense Technology, 2009.

[3] Guo Lu, Kang Peng. Application of AHP on Ship to Air Missile Weapon System Overall Schemes Evaluation, Modern Defence Technology, 35(5), pp:24-28, 2007.

[4] Meng Ke, Improvements on Decision Making Model of Multi-Attribute Intelligent Grey Target Based on Interval Number and Application, Fire Control and Command Control, 38(5), pp:98-101, 2013.

[5] Peijun Guo, Hideo Tanaka. Decision making with interval probabilities [J]. European Journal of Operational Research, 203, pp: 444-454, 2010.

[6] Guo Hui, Xu Hao-jun, Liu Ling, Threat assessment for air combat target based on interval TOPSIS. Systems Engineering and Electronics. 31(12), pp:2914-2917, 2009. 